\title{
Analyzing Social Media Discourse An Approach using Semi-supervised Learning
}

\author{
Álvaro Figueira ${ }^{1}$ and Luciana Oliveira ${ }^{2}$ \\ ${ }^{1}$ CRACS/INESC TEC, University of Porto, Rua do Campo Alegre, Porto, Portugal \\ ${ }^{2}$ CICE/ISCAP \& INESC TEC, Polytechnic of Porto, Porto, Portugal
}

Keywords: Social Media Publications, Text Mining, Automatic Categorization, Higher Education Sector, Strategic Benchmarking.

Abstract: The ability to handle large amounts of unstructured information, to optimize strategic business opportunities, and to identify fundamental lessons among competitors through benchmarking, are essential skills of every business sector. Currently, there are dozens of social media analytics' applications aiming at providing organizations with informed decision making tools. However, these applications rely on providing quantitative information, rather than qualitative information that is relevant and intelligible for managers. In order to address these aspects, we propose a semi-supervised learning procedure that discovers and compiles information taken from online social media, organizing it in a scheme that can be strategically relevant. We illustrate our procedure using a case study where we collected and analysed the social media discourse of 43 organizations operating on the Higher Public Polytechnic Education Sector. During the analysis we created an "editorial model" that characterizes the posts in the area. We describe in detail the training and the execution of an ensemble of classifying algorithms. In this study we focus on the techniques used to increase the accuracy and stability of the classifiers.

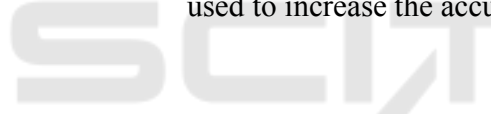

\section{INTRODUCTION}

The undeniable growth of social media environments has been introducing profound changes in society and in the communication management landscape. Though social media impacts are still subject of research in a wide variety of fields, in what organizations are concerned, two main aspects are consistently revealed throughout literature: the newly empowered role of millions of social media users, co-creators, active voices and active influencers, which organizations fail to understand and engage with, and the fact that organizations are still struggling with the development of a social media strategy and budget, thus mismanaging the potential and barriers presented by the new consumer and by social networks in general.

In fact, organizations are rushing into social media networks following the worldwide trend to create a social presence in multiple channels, reaching for and aiming at mediatization, without previously defining a clear strategic approach, which should, for instance, be built upon clear insights on their target audience and an editorial plan/calendar, that can foster the achievement of the overall business objectives. Nevertheless, when adopting social media, organizations are, in fact, allocating time, effort, skills, human resources and technology and this raises the constant need to measure the return on these investments (ROI) and legitimize them in the context of organizational development.

However, how can organizations attempt to measure the efficiency and return on investments on a social media approach that has not been strategically designed/aligned and is a set of unarticulated processes and situational messages?

On top of the absence of a strategic alignment between social media approaches and organizational goals/performance, organizations are also lacking strategically relevant social media monitoring methods.

The social media analytics provided by the thousands of free/commercial web based applications are able to provide some interesting and valuable insights, but fail to support a relevant and insightful benchmarking process. Social media monitoring has been turned into a process where organizations are on the run to acquire, for instance, more fans that their competitors', more likes and, in 
some cases, more positive feedback, where sentiment analysis is part of the equation.

However, a well sustained strategic benchmarking assessment that provides information on the current implicit/explicit strategy and knowledge on how to further develop it, it's still lacking.

Therefore, the assessment of social media performance should rather be built upon the most relevant business areas, as a key priority for organizations that aim at turning social networks into true business assets.

As a result, we present a persistent monitoring methodology built upon benchmarking methods, which rely heavily on the identification and analysis of a set of strategically relevant editorial areas that can foster organizational performance. According to the proposed methodology, organizations are propelled to focus not only on the traditional Social Media key performance indicators, but to incorporate them on a deeper editorial analysis that may allow them to gain medium and long term competitive advantage.

This article is organized as follows: in the next section (2) we describe our methodology, focussing on the development of an editorial model that will allow us to categorize social media discourse. In section 3 we present a case study where we applied the methodology described. In this section we elaborate on the type of data to retrieve from social networks, on the ensemble of chosen algorithms, and how to improve their accuracy as a whole. We finish the section with a discussion on the techniques used. In the last section (5) we present our conclusions.

\section{METHODOLOGY}

Our proposed approach is based on a 5-stage method.

On a first stage we verify which are the social networks being used by the organization under scope (main agent) and by its direct competitors, on the Higher Public Polytechnic Education Sector (HPPES). In order to obtain a comprehensive analysis, all social networks should be included and a "representative" time frame should also be chosen. The time frame that we believe to be the most suitable is a one-year time frame, because it is in itself cyclic and more likely to encompass a full cycle of communication and product/service events. After deciding on the social networks and on the ideal time frame, all of the agent's and agents' competitors messages must be collected (posts, tweets, etc.).

Hence, the second stage of the methodology consists of gathering all the information about all the sector's agents' activities on social media on: messages' content, the sector audience (type of audience, number of fans, followers, etc.) and on the corresponding responsiveness (likes, shares, comments, retweets, etc.).

The third stage of the methodology consists of identifying which are the most relevant areas of intervention on social media, which we designate by editorial areas. When this is not previously set by the agent or is not very clear (i.e. a lack of a formal content strategy is visible) a first human analysis and classification of social media messages is required. In this case, a small sample of messages manually classified by a communication professional that determines, in terms of editorial areas, which is the purpose of each message. When doing so, a set of guiding principles is considered for the HPPES in particular, which lead to the construction of the editorial model presented on section 3.2: (a) the organizations/enterprise institutional (brand) needs towards the diversity of stakeholders in its social media networks; (b) the specifics of its product(s)/service(s); (c) the need to balance between institutional and transactional needs in order to maintain reputation and ensure economic survival; (d) a multi-channel wide holistic approach to communication management which facilitates integrated messages to take the most advantage of each of the social networks being used; and the dialogical nature that is intrinsically linked to social media environments.

These principles should be considered in the definition of an editorial model for every economy sector. For instance, for common secondary sector organizations the main editorial areas could focus on: (a) core product/service advertisement; (b) availability of additional services and/or available customer support; (c) brand reputation (maintenance / reinstatement / re-branding, etc.) and (d) relationship essential in every social media channel, regardless of the economy / business sector.

The fourth stage of the methodology consists, then, on the categorization of all the messages retrieved from all the social networks against the categories' of the devised model. Although this is a complexity linear problem $(\mathrm{n} \times \mathrm{m})$, it might be too demanding to be done by hand. In fact, a categorization of $n$ posts into $m$ categories is a far too heavy endeavour to be performed by humans if we are consider thousands of posts and more than two categories (the common situation). Therefore, 
for this stage we use an ensemble of tuned algorithms which are trained to classify posts in a first moment, and that are presented the full sample to classify, on a second moment. To mitigate the occurrence of disputes in classifying a post according to several possibilities, performed by different algorithms, we take the majority of the classifications and we leave unlabelled the posts for which it was not possible to reach a majority.

Finally, the fifth stage of the methodology consists on delivering a sectorial performance and strategic benchmarking, aimed at social media organizational success.

We begin by building a performance benchmarking analysis based on social media key performance indicators (KPI), such as 'likes', 'shares', 'comments', 'retweets', etc., using a weighted scale, in order to measure audience response to messages. We then measure each market's agent's audience size (i.e. the number of 'fans', 'followers', etc.) and total communication efforts (i.e. number of messages sent to social networks).

These performance benchmarks allow us to build a performance perceptual map of the sector, in which we relate audience size, audience response and the agents' efforts.

The strategic benchmarking analysis is, then, built on top of the previous analysis, adding the social media strategies per agent (i.e. the different combinations of intensities of editorial areas), thus allowing to identify and examine the most efficient communication strategies, which are enabling high performing agents to be successful in social media.

\section{CASE STUDY}

The present case study was conducted on the Portuguese Higher Public Polytechnic Education Sector (HPPES), using the previously presented methodology.

A total of 137 agents were considered, which included polytechnic schools integrated into polytechnic institutes and polytechnic schools integrated into universities. The number of agents was then reduced to 94 in order to include only the schools providing educational services, disregarding the polytechnic institutes and universities (managing entities).

During the data collection phase we measured the HPPEI's social media networks adoption rates, in order to include the most relevant communication channels (social media), and only those that had been in use at least since the 1st of September 2013 with the intent of extending the analysis to a full school year (up to September 2014). In order to support the research in reliable sources, the study considered only the social media websites mentioned on the HPPEI's official websites. This method aimed to ensure that the social media websites under analysis were actually managed by the HPPEI, instead of other internal or external stakeholders, such as students, employees (administrative or faculty) or alumni on their own. According to these criteria, 43 agents were included in the study. Facebook proved to be the most representative social media website, with an adoption rate of $64 \%$ among all agents, as illustrated in figure 1.

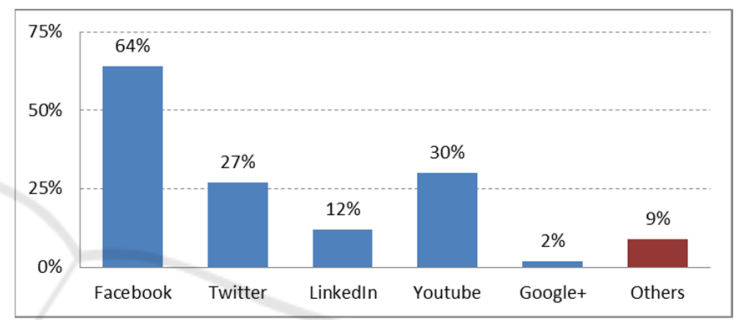

Figure 1: Social media adoption rate by agents.

In this article we focus our analysis only on Facebook, once it is the most relevant network in the sector and results adequate enough to provide evidence on the implementation of the proposed methodology.

\subsection{Retrieval of Facebook Posts}

The following stages consisted of retrieving and classifying all messages posted by HPPEI on Facebook. We used two methods: an in-house made system, specially built for the purpose using the available Facebook API and a third-party software for collecting information from Social Networks. From an initial list of the relevant agent Page Id's, the two systems accessed the posts retrieving the following fields:

List 1: Fields collected from Facebook posts.

$\begin{array}{ll}\text { 1) } & \text { PostId } \\ \text { 2) } & \text { Message } \\ \text { 3) Link } \\ \text { 4) Name } \\ \text { 5) Description } \\ \text { 6) Caption } \\ \text { 7) \#Likes } \\ \text { 8) \#Comments } \\ \text { 9) \#Shares }\end{array}$

1) PostId

2) Message

4) Name

5) Description

7) \#Likes

9) \#Shares 
The two systems retrieved the same number of posts (15.444), during the entire school year, which consolidated our confidence about the validity of the returning set.

\subsection{Editorial Model for the HPPES}

As previously mentioned, if the identification of the editorial areas for a specific agent/market is not yet set, is incomplete or is only implicit, a small sample of social media messages should be ran through a communication professional, so an efficient and comprehensive editorial model can be built.

Specifically concerning the HPPES, some editorial areas are straight forward and some were added after a manual classification of a small sample of messages.

In any case, the previously identified guiding principles for the design of a social media editorial model where applied and tuned to the HPPES and we considered the following: (a) the heavy HEI's mission towards society and the great diversity of organizational stakeholders (students, faculty, staff, employers, partners, research centres, etc.); (b) the specifics of the educational service (a co-produced service); (c) a multi-channel wide holistic approach to communication management; (d) the need to balance between organizations' institutional and transactional needs in order to ensure their competiveness and financial survival; and (e) the dialogical nature that is intrinsically linked to social media environments.

The editorial model designed for this specific case includes the seven main editorial areas that we believe to have the highest impact on organizational performance:

Education: messages are aimed at promoting or providing information about the educational offer, mainly higher education courses, but also include complementary internal or external training;

Research: messages are aimed at promoting internal research results, mainly obtained by faculty members as inputs to the organization's areas of expertise, but also include research results from other sources with impacts on those same areas. Also includes information and call for participation on congresses, seminars and other scientific meetings held internally or externally;

Society: this category builds upon the, so called, "third mission" of HEI, which is aimed at engaging with industry and other cultural and social groups, encompassing exchanges with society at large. Messages in this category include the promotion of and/or information on: knowledge and technology transfer, patents, organizational partnerships and contracts, demonstrations, exhibitions and showcases conducted by faculty members or students, and also messages promoting employability through streaming placement offers and career opportunities;

Identity/Brand: mainly aimed at the construction, development and maintenance of the organizational image and reputation, fostering distinctiveness and the development of a corporate persona. Messages consists mainly on promoting and/or informing about the corporate persona character and include references to CSR initiatives, institutional events (such as celebrations, awards, tributes and graduation ceremonies), students, faculty and staff honorable mentions and representation activities in external fairs and exhibitions;

Administration: is aimed at partially extending the internal administrative communication with internal publics into social media, but also attending administrative needs towards external stakeholders. It informs about deadlines and administrative processes, procedures and admissions, but it also promotes and informs on organizational support services (goals, contacts, working hours, etc.);

Relationship: this category builds upon the previously mentioned dialogical nature that is intrinsically linked to social media environments and aims to foster conversation, boost emotional connection the organization and its stakeholders, requiring opinions, introducing current internal, external, societal or academic issues with which publics can relate to. Messages in this category tend to present lower levels of formality in order to propel interactions and may introduce greetings, humor, sympathy and motivation.

Information: messages in this category are aimed at enhancing HEI's role in fostering citizenship, mainly among students, thus streaming external social, economic, political and cultural relevant information, news, regulations and events that may or may not be close to the schools adjoining scientific areas.

\subsection{Automatic Categorization}

Our next step was to perform the classification of the 15444 posts according to our editorial model, listed in the previous section. This is a time demanding task to be done by hand and impossible to be undertaken on the-fly if done exclusively by humans. Therefore, we propose an automatic method to categorize the posts based on the conjunction of several of the most recent and promising algorithms for text classification or categorization.

Although several text classifiers have been proposed over the last decades, nowadays this 
research topic is again gaining a lot of interest from the research community. The main reason is that much research is being focused on social networks due to the abundance of interesting data to work with. In particular, texts posted in social networks have special properties that haven't been considered in previous research and in the devised algorithms (e.g. very short texts, abundance of smileys, inclusion of links, many punctuation signs). These special characteristics pose new problems and make text classification, again, a very difficult task, and prone to failure. Nevertheless, research has been incorporating these new features and consistently creating better classification models, especially for classification under supervised training.

For this step we decided to use six of the most promising, and prominent, classifiers:

Support Vector Machines. Linear SVMs are a machine learning algorithm (Cortes, 1995) based on a geometric method that tries to separate two classes through an hyperplane, picking the one that maximizes the margin between the two classes. More recently, this method was evolved (Crammer, 2002) to deal with a multiple number of classes. We used the Multi-class SVM lib for this analysis.

Random Forests. RFs were created (Breiman, 2001) to overcome the overfitting effect of the decision trees. Within this method multiple decision trees are created during training time, and the mode of the resulting class is the presented output.

LogiBoost (Friedman, 2000). This algorithm belongs to a larger category of boosting algorithms which comprehend AdaBoost, LPBoost and some others, all based on a common framework called AnyBoost (Mason, 2000). Generically, the boosting algorithms try to reduce variance and pre-training effects in supervised learning by re-weighting a set of classifiers according to the rule: weak classifiers should gain weight and strong classifiers should lose weight. The LogiBoost is implemented in several regression and classification packages. We used the one implemented in "caTools" for R.

K-Nearest Neighbours (Altman, 1992). Although being one of the simplest, machine learning algorithm, it is still very useful because of it wide range of applicability. The algorithm relies on the previous classification of the neighbors to each training data, classifying according to the majority up to the defined $\mathrm{k}$ elements. The training data is presented in a vector space model and all trained examples are vectors in that multidimensional space.

MultiLayer Perceptrons. The "perceptron" is an algorithm, in Machine Learning theory, that is able to classify an input vector using a linear prediction function, which combines a set of computed weights to the vector parameters (Freund, 1999). When it is needed to solve non-linear problems we need more than a layer of perceptrons. Typically, multi-layer perceptrons (MLP), use sigmoide function as an activation function.

Deep Neural Networks. This type of algorithms (Collobert, 2008) are based on the concept of pretraining a multi-layered feedforward neural network, one layer at a time, treating each layer as an unsupervised restricted Boltzmann machine, and then using supervised backpropagation for finetuning the neural net.

Deep learning algorithms are based on an underlying assumption that observed data is generated by the interactions of a multitude of different factors on different levels. Deep learning assumes that these factors can be organized into multiple different levels of abstraction. Therefore, varying the number of layers and of layer sizes can provide the needed amounts of abstraction (Bengio, 2013).

All the algorithms were used through public and open source libraries ("caret" and "h2o"), available for the R programming language.

\subsubsection{Training Phase}

First, we trained manually 350 posts according to the derived model. As in the sample set there were blank posts (with no text message) we considered those to be included in a special category which we labeled as 0 (zero). The manual classification produced a total coverage of the $7+1$ categories, but not equally balanced, as illustrated in Figure 2.

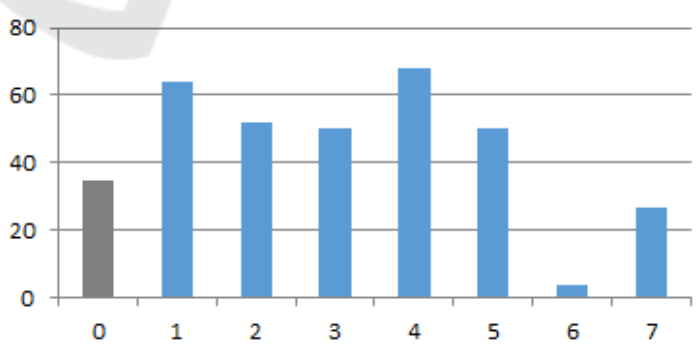

Figure 2: Label count for the 350 posts.

We then computed the respective accuracy of the automatic classification. For this we used a confusion matrix to report the number of false positives, false negatives, true positives, and true negatives. We used a standard formula (1) for computing the accuracy.

$$
\text { Accuracy }=\frac{\sum \text { True positives }+\sum \text { True negatives }}{\text { Total population }}
$$


As a second step, we gave the classifiers a bigger set of 512 manually classified posts for retraining, and recomputed the new accuracy. The new set, similarly to the first one, has a full coverage of the seven categories plus one, for blank posts, labelled as zero. We illustrate in Figure 3 the comparison of the two classifications: of the 350 and of the 512 posts, according to the number of labels. In Figure 3 the categorization of the 350 posts is represented by a dashed line and the 512 posts by a solid line. Categories are distributed along the $\mathrm{x}$ axis.

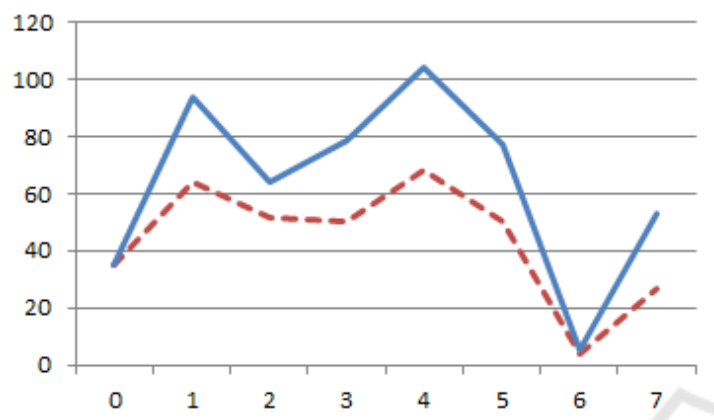

Figure 3: Label count for the 350 and the 512 posts.

It is easy to see that category 6 is still problematic due to its reduced number of posts. Apart from that consideration, we may also observe a tendency for a proportional increase in the number of posts in the remaining categories, when expanding the analysis from 350 to 512 posts.

We then computed the accuracy for the new set. Every method had an accuracy increase, but the total average for this metric, in the new training, was an improvement of only $3 \%$, over the 6 techniques. Therefore, we didn't feel in the need to classify more posts manually.

On the other hand the absolute value for the accuracy was still low (circa 55\%).

Maintaining our view to classify the posts relying only of the post itself and on the features associated with each post, we augmented List 1 presenting more information to the classifiers (information that was already retrieved during post collection, but not used):

List 2: Extra fields collected from Facebook posts.
10) From
11) Date
12) Hour
13) Type
14) Status type
15) Link
16) Name
17) Story

Therefore, we had now 17 fields, possibly some of them with no values.

We tried various approaches to use all the text in the fields to help the classifiers, and combinations of several texts. For examples, we tried:

1) Message or Description

2) Message, Name, Description, Caption and Story

3) Message, Name Description, Caption, Story and link domain

4) All the 17 fields described in List 1 and 2, and using a "link explosion" strategy.

We mean by "link explosion" the separation of each term in an URL that is joined to another term by a slash, by punctuation signs, or by the protocol's name.

\subsubsection{Classification}

We then executed again the trained algorithms on the 512 sample data using all the approaches. We computed the accuracy, and found find out that approach 4 delivers the best result.

In Table 1 we can see that there is a $3 \%$ improvement from expanding the training set to 512 samples, and that, using all the 17 fields as text features, results in a significantly better accuracy.

To assess the stability of the method we performed a 10 -fold cross validation, with the input data averagely distributed, throughout the whole sample (the 512 posts) and then we computed the respective accuracy (using formula 1) in each pass, for the whole ensemble of classifying algorithms.

Table 1: Accuracy during the training phase.

\begin{tabular}{|l|r|r|r|r|}
\hline Accuracy & $\mathbf{3 5 0}$ & $\mathbf{5 1 2}$ & Diff & 512-all \\
\hline \hline SVM & 0.3264 & 0.3553 & 0.0289 & 0.4523 \\
\hline RF & 0.5347 & 0.5866 & 0.0519 & 0.6862 \\
\hline LB & 0.6044 & 0.6507 & 0.0463 & 0.7243 \\
\hline KNN & 0.4722 & 0.4803 & 0.0081 & 0.5451 \\
\hline MLP & 0.5844 & 0.606 & 0.0216 & 0.7703 \\
\hline H2ODL & 0.5781 & 0.6031 & 0.025 & 0.6527 \\
\hline \hline Average & 0.5167 & 0.547 & 0.0303 & 0.63848 \\
\hline
\end{tabular}

The above table represents just the first run of the training. We list below, in Table 2, the results of the 10 -fold cross validation.

Table 2: Accuracy of the aggregation for the six algorithms in each of the 10 runs.

\begin{tabular}{|c|c|}
\hline 1 & 0.63848 \\
\hline 2 & 0.71432 \\
\hline 3 & 0.75420 \\
\hline 4 & 0.65130 \\
\hline 5 & 0.70034 \\
\hline 6 & 0.65470 \\
\hline 7 & 0.65382 \\
\hline 8 & 0.72943 \\
\hline 9 & 0.73342 \\
\hline 10 & 0.62832 \\
\hline \hline Average & 0.68583 \\
\hline
\end{tabular}


We ended up with an average accuracy above $68 \%$, which seemed a fair base for classifying the whole set of posts.

We then run the whole set of 15444 posts on our 6 trained classifiers to obtain a predicted category for each post, by each technique. Finally, we used the prevailing category of these six techniques as the final result, i.e., we used the prevailing category of the sixset as the final predictive category. When there was no mode, the post was labelled with a zero. The number of posts per category is illustrated in Figure 4.

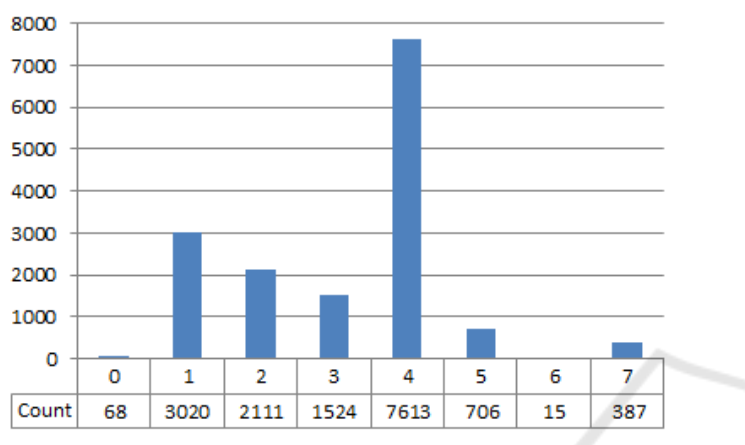

Figure 4: Final label count for the 15444 posts.

As we can see category 6 remained with very few posts (15) and less than $1 \%$ were unclassified posts (labelled as zero). Apart from Logiboost, every classifier could reach some category for every post. Logiboost failed to categorize 5055 posts (about $1 / 3$ of the total of posts). All in all, 15376 posts (i.e., more than $99 \%$ ) were successfully given some label.

\subsubsection{Strategic Benchmarking for Business Intelligence}

Having the posts categorized in each of the seven categories defined in our model allows us to build a statistics model and parameters to assess the effort and gain with each message. For instance, we can compare the number of posts in each category for every competitor and its return in the form of Likes, Shares and Comments (eventually with different weights). In a previous study (Oliveira and Figueira, 2015) it was shown that some competitors have centralized strategies whilst others have decentralized or hybrid strategies, according to the amount of effort per editorial area. Such research outputs and methodology are typically framed in strategic benchmarking processes that rely heavily on business intelligence skills. In fact, the ability to handle large amounts of unstructured data, to help identify and develop new strategic business opportunities and the identification of fundamental lessons among competitors are essential to the formation of well sustained medium / long term decision making processes.

\subsubsection{Discussion and Notes}

The "link explosion" strategy increased the accuracy of the classifiers around $2 \%$. The use of all collected features associated with each post leveraged the accuracy in about $7 \%$. All the text was concatenated and transformed into a confusion matrix of posts and terms. The TF and TF-IDF metrics were used to try to discover relevant words to the classification. This procedure resulted in a slightly better results for TF but not relevant, as neither were conclusive about its discriminatory power.

Before the training phase the more sparse features derived from the message of each post were removed, in order to obtain a maximum of 0.999 of sparsity. A 0.99 of sparsity was allowed for features derived from the link explosion.

Curiously, LogiBoost had the best accuracy for the 512 trained set, despite not being able to classify all the posts. The Multi-Layer Perceptron, was the second best during training, and became first when all features were used. Moreover, this algorithm was capable of classifying all the posts. The accuracy assessment used a 10-fold cross validation, implemented through the "caret" library. Whenever a classifier was trained using the caret library, a cross validation was used and, for each of the 10-fold was computed the accuracy and the kappa index. When we used the "h2o" library the accuracy was computed using a manually built, but standard, implementation of the metric.

In order to improve the categorization, we tried the selection of disjoint sets of words of features for every class, ie, words that are used in just one category. We also tested the removal of sparse features which had high correlation and the removal of features with high variance. All in all, the improvement was quite small which probably is due to the fact that most of the algorithms are based on

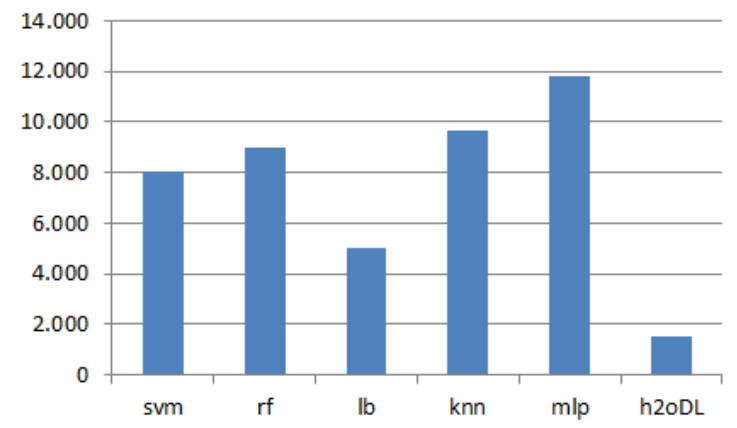

Figure 5: Correct assigned labels per algorithm according to the final label produced by the ensemble of classifiers. 
trees, which already do select relevant features during training. Finally, we must stress that the Multi-Layer Perceptron was the algorithm that more often categorized the posts as the majority of the ensemble of classifiers did (cf. Figure 2).

\section{CONCLUSIONS}

In this article we presented a model for analysing social media discourse by categorizing the messages posted in social networks according to a $n$-category model, created during analysis. Posts from an entire year are collected and a small amount is used to build the editorial model. Another sample was used for training. In our case study, the accuracy did not increase the classifying procedure with a sample bigger than $3 \%$ of the total data. In the case study we used six of the most well-known algorithms to perform the categorization. We showed how to improve their classifying accuracy by using the features associated with each post, and how to fine tune the categorization parameters. The resulting accuracy of the method was increased from $51 \%$ up to $68 \%$. After the ensemble of algorithms were trained the whole sample was ran through it.

\section{ACKNOWLEDGMENTS}

This work is supported by the ERDF - European Regional Development Fund through the COMPETE Programme (operational programme for competitiveness) and by National Funds through the FCT (Portuguese Foundation for Science and Technology) within project «Reminds/ UTAPICDT/EEI-CTP/0022/2014.

\section{REFERENCES}

Altman, N. S. (1992). "An introduction to kernel and nearest-neighbor nonparametric regression". The American Statistician 46 (3): 175-185.

Bengio, Y.; Courville, A.; Vincent, P. (2013). "Representation Learning: A Review and New Perspectives". IEEE Transactions on Pattern Analysis and Machine Intelligence 35 (8): 1798-1828.

Breiman, Leo (2001). "Random Forests". Machine Learning 45 (1): 5-32.

Cortes, C.; Vapnik, V. (1995). "Support-vector networks". Machine Learning 20 (3): 273.

Freund, Y.; Schapire, R. E. (1999). "Large margin classification using the perceptron algorithm" (PDF). Machine Learning 37 (3): 277-296.
Friedman, J., Hastie, T., and Tibshirani, R. (2000). Additive logistic regression: a statistical view of boosting. Annals of Statistics 28(2): 337-407.

Koby Crammer and Yoram Singer. 2002. On the algorithmic implementation of multiclass kernel-based vector machines. J. Mach. Learn. Res. 2 (March 2002), 265-292.

Llew Mason, Jonathan Baxter, Peter Bartlett, and Marcus Frean (2000); Boosting Algorithms as Gradient Descent, in S. A. Solla, T. K. Leen, and K.-R. Muller, editors, Advances in Neural Information Processing Systems 12, pp. 512-518, MIT Press.

Oliveira, L., Figueira, A. (2015). Benchmarking analysis of social media strategies in the Higher Education Sector. In Proceedings of Conference on ENTERprise Information Systems (CENTERIS'16). Vol64:779786.

Ronan Collobert and Jason Weston. (2008). A unified architecture for natural language processing: deep neural networks with multitask learning. In Proceedings of the 25th international conference on Machine learning (ICML '08). ACM, New York, NY, USA, 160-167.

Rud, Olivia (2009). Business Intelligence Success Factors: Tools for Aligning Your Business in the Global Economy. Hoboken, N.J: Wiley \& Sons. ISBN 978-0470-39240-9.
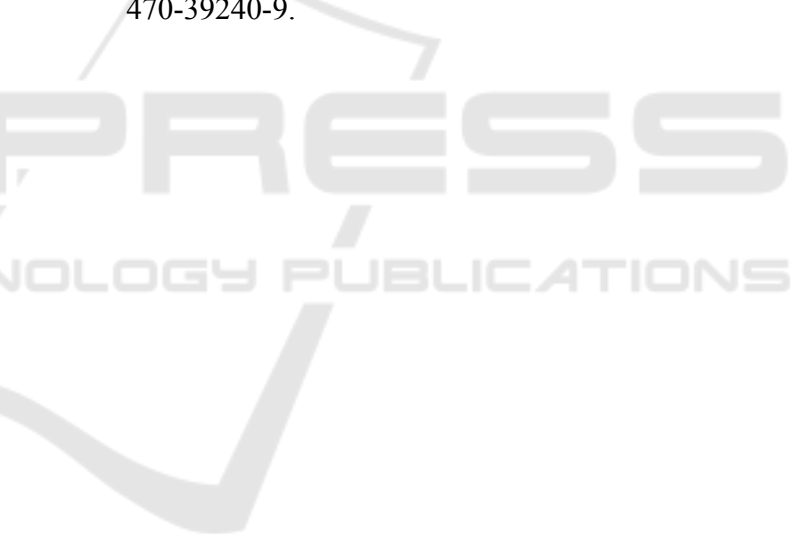